\title{
Effect of Farnesyltransferase Inhibitor R115777 on Mitochondria of Plasmodium falciparum
}

\author{
Young Ran Ha', Bae-Geun Hwang'2, Yeonchul Hong ${ }^{3}$, Hye-Won Yang ${ }^{3}$, Sang Joon Lee ${ }^{2, *}$ \\ ${ }^{1}$ Division of Integrative Bioscience and Bioengineering, Center for Biofluid and Biomimic Research, Pohang University of Science and Technology, \\ Pohang 790-784, Korea; '2Department of Mechanical Engineering, Center for Biofluid and Biomimic Research, Pohang University of Science and \\ Technology, Pohang 790-784, Korea; ${ }^{3}$ Department of Parasitology, Kyungpook National University School of Medicine, Daegu 700-422, Korea
}

\begin{abstract}
The parasite Plasmodium falciparum causes severe malaria and is the most dangerous to humans. However, it exhibits resistance to their drugs. Farnesyltransferase has been identified in pathogenic protozoa of the genera Plasmodium and the target of farnesyltransferase includes Ras family. Therefore, the inhibition of farnesyltransferase has been suggested as a new strategy for the treatment of malaria. However, the exact functional mechanism of this agent is still unknown. In addition, the effect of farnesyltransferase inhibitor (FTIs) on mitochondrial level of malaria parasites is not fully understood. In this study, therefore, the effect of a FTI R115777 on the function of mitochondria of $P$. falciparum was investigated experimentally. As a result, FTI R115777 was found to suppress the infection rate of malaria parasites under in vitro condition. It also reduces the copy number of mtDNA-encoded cytochrome $c$ oxidase III. In addition, the mitochondrial membrane potential $\left(\Delta \Psi_{\mathrm{m}}\right)$ and the green fluorescence intensity of MitoTracker were decreased by FTI R115777. Chloroquine and atovaquone were measured by the mtDNA copy number as mitochondrial non-specific or specific inhibitor, respectively. Chloroquine did not affect the copy number of mtDNA-encoded cytochrome $c$ oxidase III, while atovaquone induced to change the mtDNA copy number. These results suggest that FTI R115777 has strong influence on the mitochondrial function of $P$. falciparum. It may have therapeutic potential for malaria by targeting the mitochondria of parasites.
\end{abstract}

Key words: Plasmodium falciparum, malaria, mtDNA, farnesyltransferase inhibitor, mitochondria

\section{INTRODUCTION}

Malaria is a worldwide public health problem because it is a medical emergency with a high risk of mortality [1]. Plasmodium falciparum causes the most dangerous form of malaria to humans [2]. It also exhibits resistance to antimalarial drugs [3]. The resurgence of malaria is partly attributed to wide distribution of drug-resistant strains [4]. The enzyme protein farnesyltransferase is an ideal drug target for $P$. falciparum [5]. Farnesyltransferase is an enzyme that belongs to the prenyltransferase group [6]. It catalyzes the posttranslational modification of intracellular signal transduction proteins by transferring the farnesyl residue of farnesyl pyrophosphate [7]. The cysteine residue, which is the transferring farnesyl residue, belongs to the car-

- Received 16 March 2015, revised 4 June 2015, accepted 5 July 2015.

*Corresponding author (sjlee@postech.ac.kr)

(C) 2015, Korean Society for Parasitology and Tropical Medicine

This is an Open Access article distributed under the terms of the Creative Commons Attribution Non-Commercial License (http://creativecommons.org/licenses/by-nc/3.0) which permits unrestricted non-commercial use, distribution, and reproduction in any

medium, provided the original work is properly cited. boxy-terminal consensus sequence called the CAAX motif [7]. The targets of farnesyltransferase include members of the Ras superfamily, which are critical to cell cycle progression [8]. For this reason, inhibitors of farnesyltransferase have effects on anti-cancer drugs and anti-parasitic agents [7]. Therefore, FTls has been recommended as a novel antimalarial drug [9].

Mitochondria have been shown to play a key role in the apoptotic process through several signaling pathways. Mitochondria control the apoptosis at several levels such as maintenance of ATP production and mitochondrial membrane potential $(\Delta \Psi \mathrm{m})$ and permeability for the release of certain apoptogenic factors from the intermembrane space into the cytosol [10]. Malaria parasites have very small mitochondrial genomes, including 3 encoded proteins, and highly fragmented ribosomal RNAs [11]. The mitochondria of $P$. falciparum enable it to adapt to the host morphologically and physiologically [12]. The energy metabolism of $P$. falciparum differs from that of the other mammalian hosts [12]. P. falciparum has a simplified metabolism with biosynthetic pathways [11]. The mitochondria of $P$. falciparum do not exhibit full oxidation of glucose to 
fuel mitochondrial ATP synthesis in the malaria parasite [11]. The mitochondrial electron transport chain (mETC) is important for pyrimidine biosynthesis [11]. The mitochondria of $P$. falciparum are promising targets for antimalarial drugs because the energy-transducing pathways are distinct in the malaria parasite [12]. Thus, the inhibition of mitochondria has been suggested as a novel strategy for malaria extermination. However, the relationship between FTIs and mitochondria function of $P$. falciparum has not yet been investigated.

In this study, the effects of FTI (Zarnestra, R115777) on the mitochondrial function of $P$. falciparum were investigated under in vitro condition. In addition, to compare the drug target, after treating chloroquine and atovaquone in vitro, the copy number of mtDNA-encoded cytochrome $c$ oxidase III was measured for each case. The function of mitochondria was examined by staining with the mitochondrial membrane potential $(\Delta \Psi \mathrm{m})$-sensitive dyes and MitoTracker.

\section{MATERIALS AND METHODS}

\section{Chemicals}

FTI was purchased from Selleck (Zarnestra, R115777, Houston, Texas, USA). Chloroquine and atovaquone were purchased from Sigma-Aldrich (St. Louis, Missouri, USA).

\section{Parasite culture}

P. falciparum (3D7 strain) was maintained in human erythrocytes (blood group $\mathrm{O}^{+}$) in RPMI 1640 (buffered with $25 \mathrm{mM}$ HEPES and $25 \mathrm{mM} \mathrm{NaHCO} 3$ ) supplemented with $10 \mu \mathrm{g} / \mathrm{ml}$ gentamycin and $370 \mu \mathrm{M}$ hypoxanthine under a gas mixture containing $5 \% \mathrm{CO}_{2}, 5 \% \mathrm{O}_{2}$, and $90 \% \mathrm{~N}_{2}$ at $37^{\circ} \mathrm{C}$ in an incubator. Parasites were diluted with $9.5 \mathrm{ml}$ of culture medium containing $0.5 \mathrm{ml}$ of sufficient non-infected human erythrocytes to yield a final hematocrit of $5 \%$ and a parasitemia of $0.5 \%$ [13].

\section{Giemsa staining}

A thin blood smear was fixed with $99 \%$ methanol for $5 \mathrm{~min}$. After fixing the slide, it was dried for $1 \mathrm{~min}$ to $2 \mathrm{~min}$. A diluted Giemsa solution (Merck, Darmstadt, Germany) was prepared prior to staining. The slide was immersed in freshly prepared Giemsa stain solution for $25 \mathrm{~min}$, rinsed with tap water, and dried. The stained malaria parasites were examined using a microscope with a $100 \times$ oil immersion objective lens.

\section{Synchronization of $P$. falciparum}

Synchronized ring-form cultures were tested to investigate the antimalarial effects. Sorbitol (5\%) was dissolved in distilled water, and passed through a Millipore filter of $0.22 \mu \mathrm{m}$ pores. Sorbitol $(9 \mathrm{ml})$ was added to $1 \mathrm{ml}$ of the culture pellet, and incubated for $5 \mathrm{~min}$ in room temperature. The supernatant was removed by centrifugation, and the pellet was washed 3 times in complete media. P. falciparum was determined to be free of schizonts by microscopic examination of the Giemsastained specimen [14]. After synchronization, ring form of $P$. falciparum were treated with each drugs for $48 \mathrm{hr}$. The infection rate in synchronized $P$. falciparum was approximately 1.8-2.0\%.

\section{MitoTracker labeling}

To measure the mitochondria mass, MitoTracker Green was treated in malaria-infected RBCs after FII R115777 treatment for $48 \mathrm{hr}$. The $9 \mathrm{ml}$ of supernatant of malaria-infected RBCs was removed by centrifuge and malaria-infected RBCs were incubated with $1 \mathrm{mM}$ MitoTracker stock solution for $30 \mathrm{~min}$ at $37^{\circ} \mathrm{C}$. After staining with a MitoTracker dye, the malaria-infected RBCs were washed in incomplete media. Fluorescence imaging techniques were employed with the excitation and emission wavelength bands centered at 490 and $516 \mathrm{~nm}$, respectively. Fluorescence was measured by a CytoFluor fluorescence multiwell plate reader (Tecan USA, Durham, North Carolina, USA). Fluorescence signal intensities were evaluated using Image J software (Image 1.46r software, U.S. National Institutes of Health, Bethesda, Maryland, USA).

\section{Determination of mitochondrial membrane potential $(\Delta \Psi \mathrm{m})$}

Variations in $\Delta \Psi \mathrm{m}$ were analyzed using 5, 5', $6,6^{\prime}$-tetrachloro-l, l', 3, 3'-tetraethylbenzimidazolcarbocyanine iodide (JC-1) (Molecular Probes, Invitrogen, Eugene, Oregon, USA), which was stained as previously described [15]. Malaria parasite infected RBCs treated with FTI R115777 for 48 hr were incubated with JC-1 reagent for $20 \mathrm{~min}$ at $37^{\circ} \mathrm{C}$. The supernatant was removed by centrifugation, and the pellet was washed 3 times in Cell-Based Assay buffer (Molecular Probes, Invitrogen). Malaria-infected RBCs were analyzed using a CytoFluor fluorescence multiwell plate reader. For healthy conditions, JC-1 forms J-aggregates in excitation and emission wavelengths of 560 and $595 \mathrm{~nm}$, respectively. For apoptotic or unhealthy conditions, JC-1 exists as a monomer at 485 and $535 \mathrm{~nm}$. Fluorescence signal intensities were measured using Image J software. 


\section{Determination of oxygen gradients}

P. falciparum was treated with FTI R115777 for $48 \mathrm{hr}$ after sorbitol synchronization. The sensor foil (SF-RPSU4, PreSens Precision Sensing $\mathrm{GmbH}$, Regensburg, Germany) was calibrated by placing a drop of sodium dithionate $\left(\mathrm{Na}_{2} \mathrm{~S}_{4} \mathrm{O}_{4}\right)$, and recording the image [16]. The oxygen concentration was evaluated using VisiSens Analytical 1 software. After recording the calibration value, the sensor foil was sterilized by $70 \%$ ethanol, and washed with autoclaved distilled water several times. Malaria-infected RBCs were placed on the oxygen-sensitive sensor foil, and data were read using a detector unit.

\section{Detection of reactive oxygen species (ROS) content by dichlorofluorescein (DCF)}

To measure ROS activity, an OxiSelect ROS assay kit (Cell Biolabs, San Diego, California, USA) was used as previously described [17]. The assay uses the diffusion of 2', $7^{\prime}$-dichlorodihydrofluorescin diacetate (DCFH-DA) into cells. DCFH-DA is deacetylated by cellular esterases to non-DCFH and rapidly oxidized to DCF by ROS. In brief, malaria-infected RBCs were pre-incubated with DCFH-DA $(100 \mu \mathrm{M})$ for $20 \mathrm{~min}$ before adding FTI R115777. A CytoFluor Fluorescence Multiwell Plate Reader (Tecan USA) was used to determine ROS production by comparing the results with a predetermined DCF standard curve (excitation $=480 \mathrm{~nm}$, emission $=520 \mathrm{~nm}$ ). Fluorescence signal intensities were measured using Image J software.

\section{Isolation of mitochondrial DNA}

P. falciparum was maintained for $48 \mathrm{hr}$ in drugs for treatment after sorbitol synchronization. The mtDNA was isolated using mtDNA Extractor CT kits (Wako pure Chemical Industries, Ltd., Osaka, Japan) following the manufacturer's instructions [18]. Malaria parasites were homogenized in ice-cold homogenization buffer provided in the kit. The homogenate was centrifuged at $1,000 \mathrm{~g}$ and $4^{\circ} \mathrm{C}$ for $1 \mathrm{~min}$. The supernatant was collected and centrifuged at $10,000 \mathrm{~g}$ and $4^{\circ} \mathrm{C}$ for $10 \mathrm{~min}$. The $\mathrm{mtDNA}$ extraction solutions in the kit were added to the pellet. mtDNA was precipitated by the addition of $300 \mu \mathrm{l}$ of sodium iodide solution and $500 \mu \mathrm{l}$ of isopropanol. The mtDNA concentrations were measured using Nano-Drop (Thermo Scientific, Wilmington, Delaware, USA).

\section{mtDNA amplification}

All oligonucleotide primers were used as described in Cunha et al. [19]. The sequences of cytochrome $c$ oxidase III (GenBank accession nos. GI8346992 and M76611) expressed from P. falciparum used in this study were Pf1 (5'-CCTGCATTAACATCATTATATGGTACATCT- $3^{\prime}$ ) and Pf2 (5'-GATTAACATTCTTGATGAAGTAATGATAATACCTT-3') [19]. PCR was conducted using Accupower PCR premix (Bioneer, Daejeon, Korea). The equivalent amount of the mtDNA were used. All procedures were performed according to the manufacturer's instructions. The amplification conditions were as follows: 1 denaturation cycle at $96^{\circ} \mathrm{C}$ for $10 \mathrm{~min} ; 30$ cycles at $95^{\circ} \mathrm{C}$ for $1 \mathrm{~min}$ and $60^{\circ} \mathrm{C}$ for 5 min; and a final extension at $60^{\circ} \mathrm{C}$ for $1 \mathrm{hr}$. All amplifications were performed in a MyGenie 96 Gradient Thermal Block (Bioneer). The PCR products were resolved with standard agarose gel electrophoresis, and visualized using ethidium bromide staining under UV light.

\section{Statistical analysis}

All statistical data were analyzed using SPSS $t$-test (IBM, Chicago, Illinois, USA) at 95\% coverage.

\section{RESULTS}

\section{Growth inhibitory activity of FTI R115777}

To elucidate the antimalarial effects of FTI R115777, P. falciparum was treated with the drug for $48 \mathrm{hr}$. Before treating drugs, parasitemia in synchronized $P$. falciparum was approximately 1.8-2.0\%. Parasitemia after drug treatment was $13.4 \pm 0.3 \%$ in the control group. FTI R115777 inhibited the proliferation of malaria parasites in a dose-dependent manner. The $\mathrm{IC}_{50}$ for FTI R115777 for $P$. falciparum 3D7 was $11.48 \mathrm{nM}$ in vitro. FTI R115777 (100 nM) inhibited the growth of parasites by $65.2 \pm$ $1.8 \%$ (Fig. 1A). Malaria parasitemias became $5.8 \%, 5,6 \%$, and $3.0 \%$ after addition of $0.05,0.1$, and $0.5 \mu \mathrm{M}$ of FTI R115777, respectively. The high-dose FTI R115777-treated $P$. falciparum group exhibited distinctive morphological features, such as pyknosis form, compared with the control group (Fig. 1A). In addition, we treated atovaquone, known to target the mitochondria, and chloroquine that does not have a specific mitochondrial effect $[20,21]$. Chloroquine and atovaquone were observed to inhibit $P$. falciparum infection as coincided with Fig. 1B and 1C. Malaria parasitemias became 6.8\%, 5.7\%, and $1.7 \%$ after addition of 10,100 , and $100 \mathrm{nM}$ of chloroquine, respectively (Fig. 1B). When $0.1,1$, and $10 \mathrm{nM}$ of atovaquone was treated, parasitemia was $7.7 \%, 5.9 \%$, and $1.2 \%$, respectively (Fig. 1C). These results showed the antimalarial effect of FTI R115777, cloroquine, and atovaquone. 


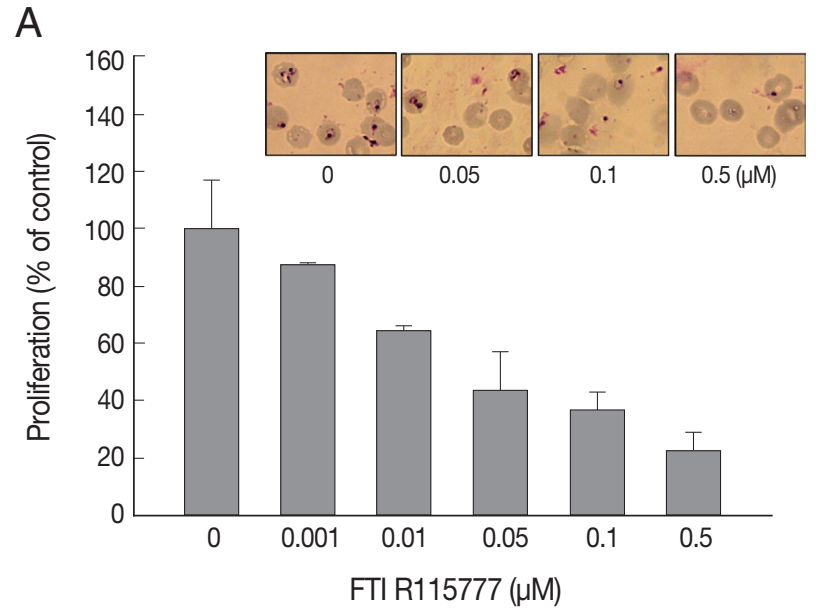

B

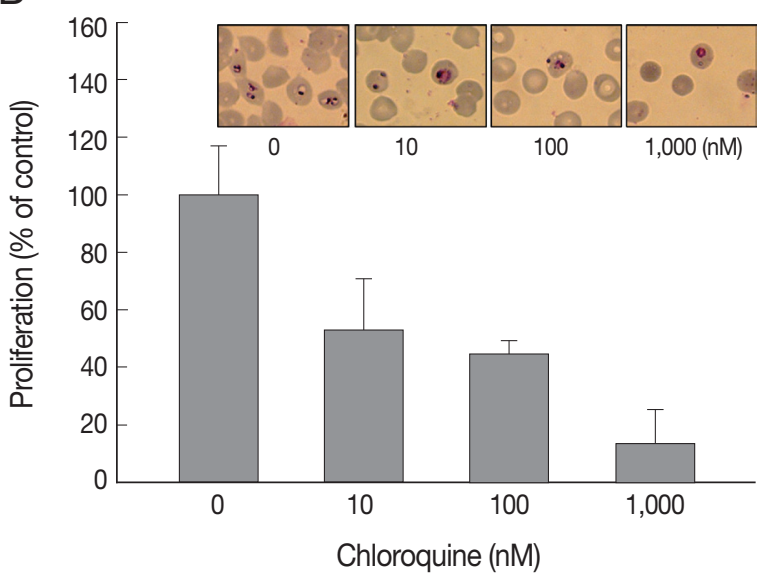

C

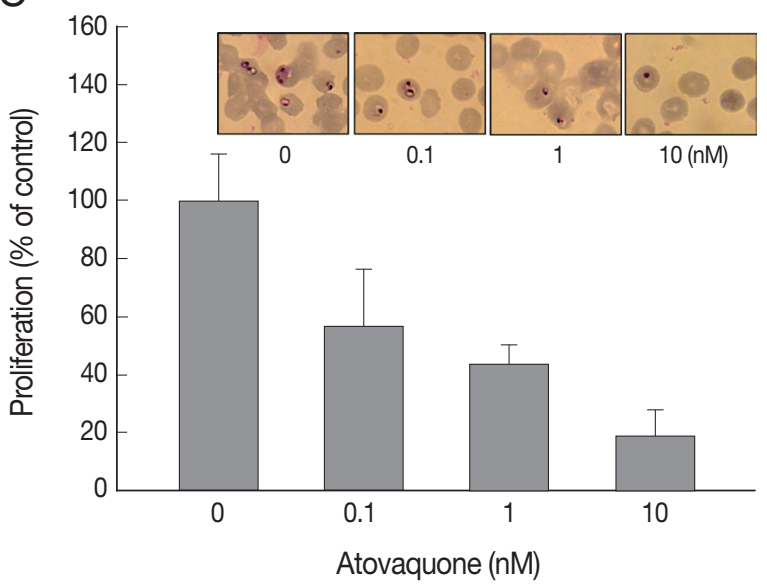

Fig. 1. Effect of 3 antimalarial drugs on the proliferation of $P$. falciparum. (A) Proliferation assay of FTI R155777-treated $P$. falciparum for $48 \mathrm{hr}$ and corresponding Giemsa staining. (B) Proliferation assay of chloroquine-treated $P$. falciparum and Giemsa staining. (C) Proliferation assay of atovaquone-treated $P$. falciparum and Giemsa staining.
Maintenance of mitochondrial membrane potential $\Delta \Psi \mathrm{m}$

Given that FII R115777 suppresses the proliferation of $P$. falciparum, we investigated whether this action works specifically through the mitochondria. As shown in Fig. 2A and B, the low-dose FTI R115777-treated group exhibited strong red fluorescence, which indicated a healthy condition. By contrast, the high-dose R115777-treated group exhibited green fluorescence because of low $\Delta \Psi \mathrm{m}$ compared with that of the control group. These results showed that mitochondrial depolarization was induced by FTI R115777.

\section{Intensity of MitoTracker green fluorescence in FTI R115777-treated malaria parasites}

To investigate the effect of FII R115777 on the mitochondrial function of $P$. falciparum, MitoTracker green fluorescence was stained in vitro. MitoTracker labels the mitochondria in live cells using $\Delta \Psi \mathrm{m}$, and can be used for multiple labeling and measuring of the depolarized mitochondrial mass $[22,23]$. The cells stained by MitoTracker were observed under fluorescence microscopy, and the fluorescence signal intensities were measured using Image J software. Fig. 2C and D clearly show that the MitoTracker green fluorescence signal decreased as the dose of FII R115777 increased. This result indicated that FII R115777 can be used as an effective target to mitochondria.

\section{Oxygen concentration of FTI R115777-treated malaria parasites}

To examine the mitochondrial activity, extracellular oxygen concentrations were measured via the ratiometric read-out of the sensor foil by VisiSens devise. The oxygen gradient of FII R115777-treated $P$. falciparum were shown on the sensor foil (Fig. 3A). As shown in Fig. 3B, the oxygen concentration of malaria-infected RBCs was higher than that of the non-infected RBCs $(P<0.05)$. However, the average oxygen concentration started to decrease slightly from the dose of $0.1 \mu \mathrm{M}$ to 1 $\mu \mathrm{M}$ FII R115777. This result implied that depolarization was mainly mediated by other contributors rather than oxygen consumption.

\section{Effect of FTI on the intracellular ROS level}

We investigated whether FTI R115777-treated P. falciparum was linked to mitochondrial ROS generation. The DCFH-DAmeasurable ROS induced by FII R115777 in the malaria parasites was measured. ROS levels notably increased in malariainfected RBCs than those in non-infected RBCs. The intracel- 

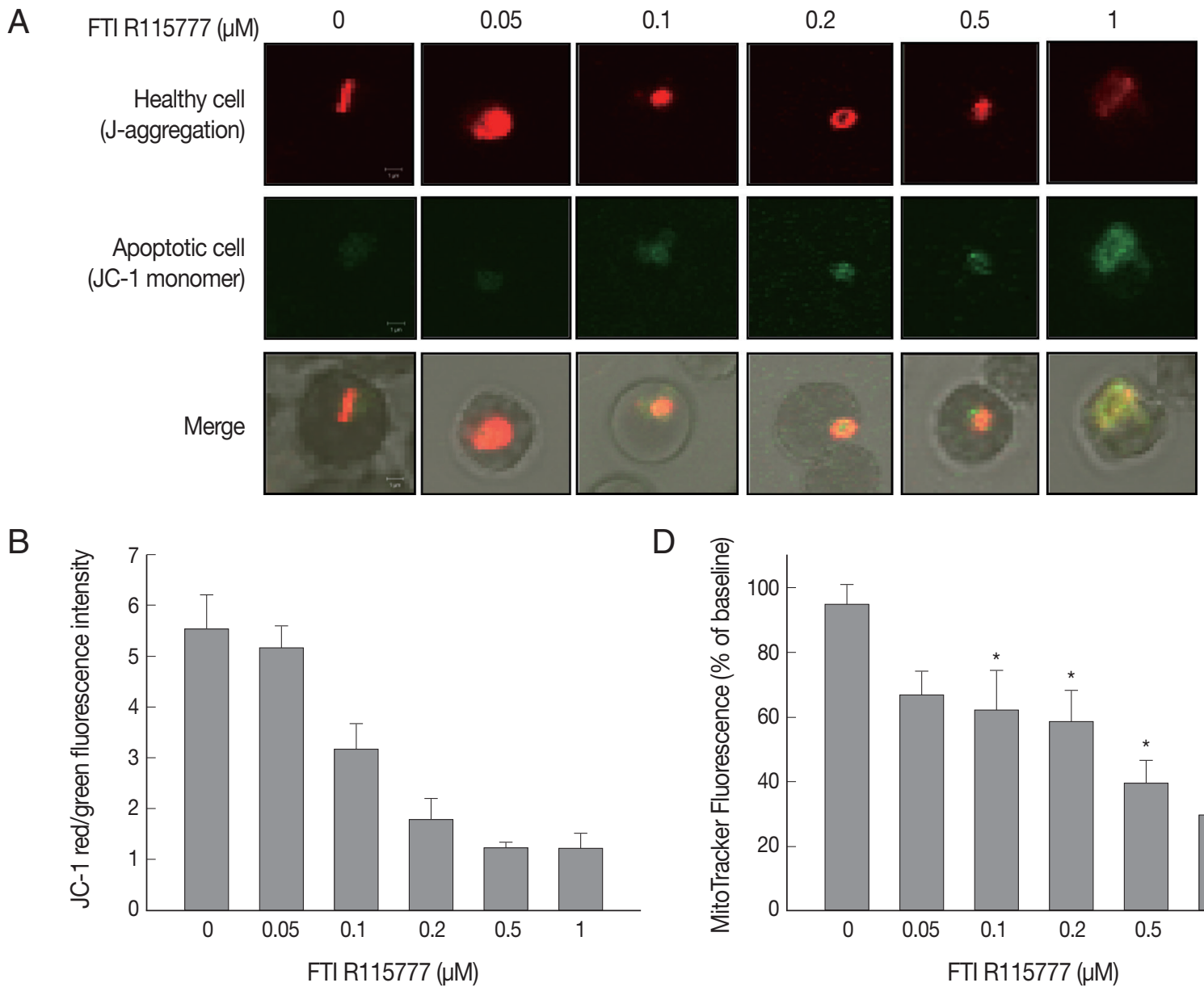

B

C
$\mathrm{D}$

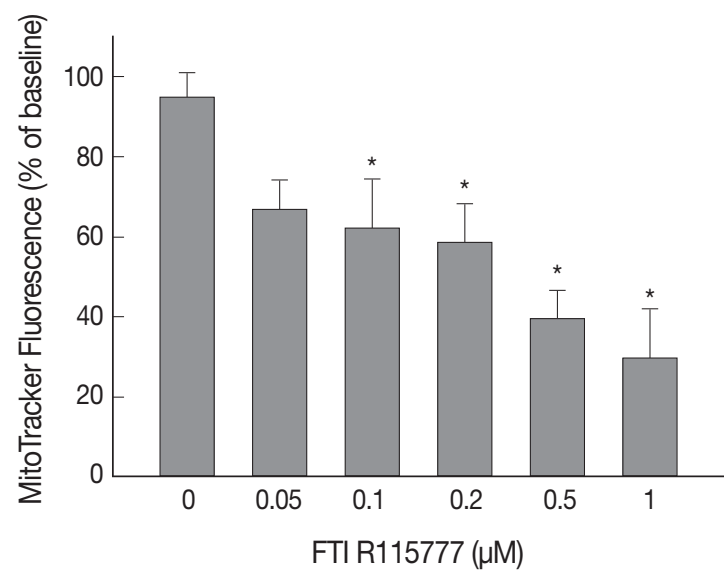

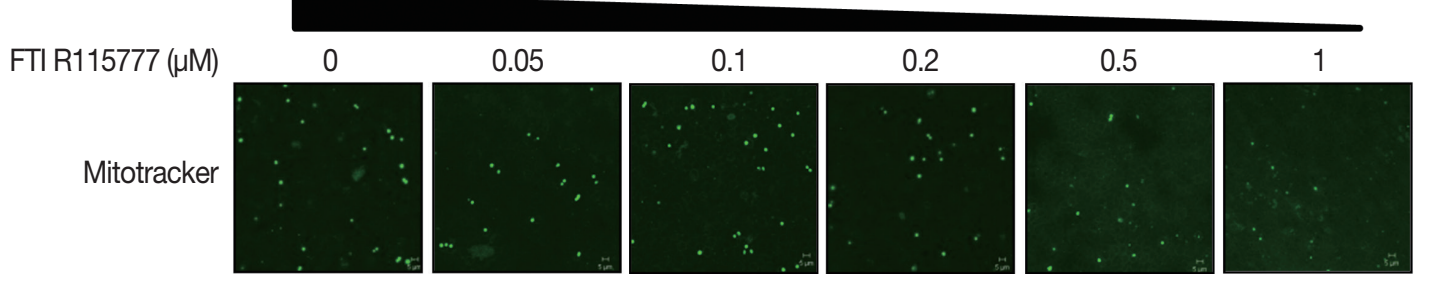

Fig. 2. Analysis of mitochondrial membrane potential $(\Delta \Psi \mathrm{m})$ after $48 \mathrm{hr}$ of $\mathrm{FTI} \mathrm{R} 115777$ treatment using the potential-dependent aggregate-forming lipophilic cation JC-1 (5, 5', 6, 6'-tetrachloro-1, 1', 3, 3'-tetraethylbenzimidazole carbocyanide iodide). (A) JC-1 staining. Red fluorescence represents mitochondria with intact membrane potential, whereas green fluorescence indicates de-energized mitochondria. (B) JC-1 red/green fluorescence intensity. (C) Immunofluorescence analysis of FTI R115777-treated P. falciparum cells for 48 hr stained with MitoTracker green. (D) Fluorescence intensity of MitoTracker green in FTI R115777-treated P. falciparum. Bars denote SD. ${ }^{*} P<0.05$.

lular ROS levels increased as the dose of FTI R115777 increased (Fig. 3C). This result indicated that the FTI R115777-induced intracellular ROS could sufficiently disrupt the mitochondrial functions. The specificity of FII R115777 could be attributed to the selective interaction with malarial mitochondria. This interaction may specifically generate ROS in malarial mitochondria, which are key factors that enable the manifesta- tion of the antimalarial drug effect of FTI R115777.

\section{Copy number of cytochrome $c$ oxidase III in FTI} R115777-treated $P$. falciparum

In this study, we measured the copy number of mtDNA-encoded cytochrome $c$ oxidase III in FTI R115777-treated P. falciparum. FTI R115777 was observed to reduce the copy number 

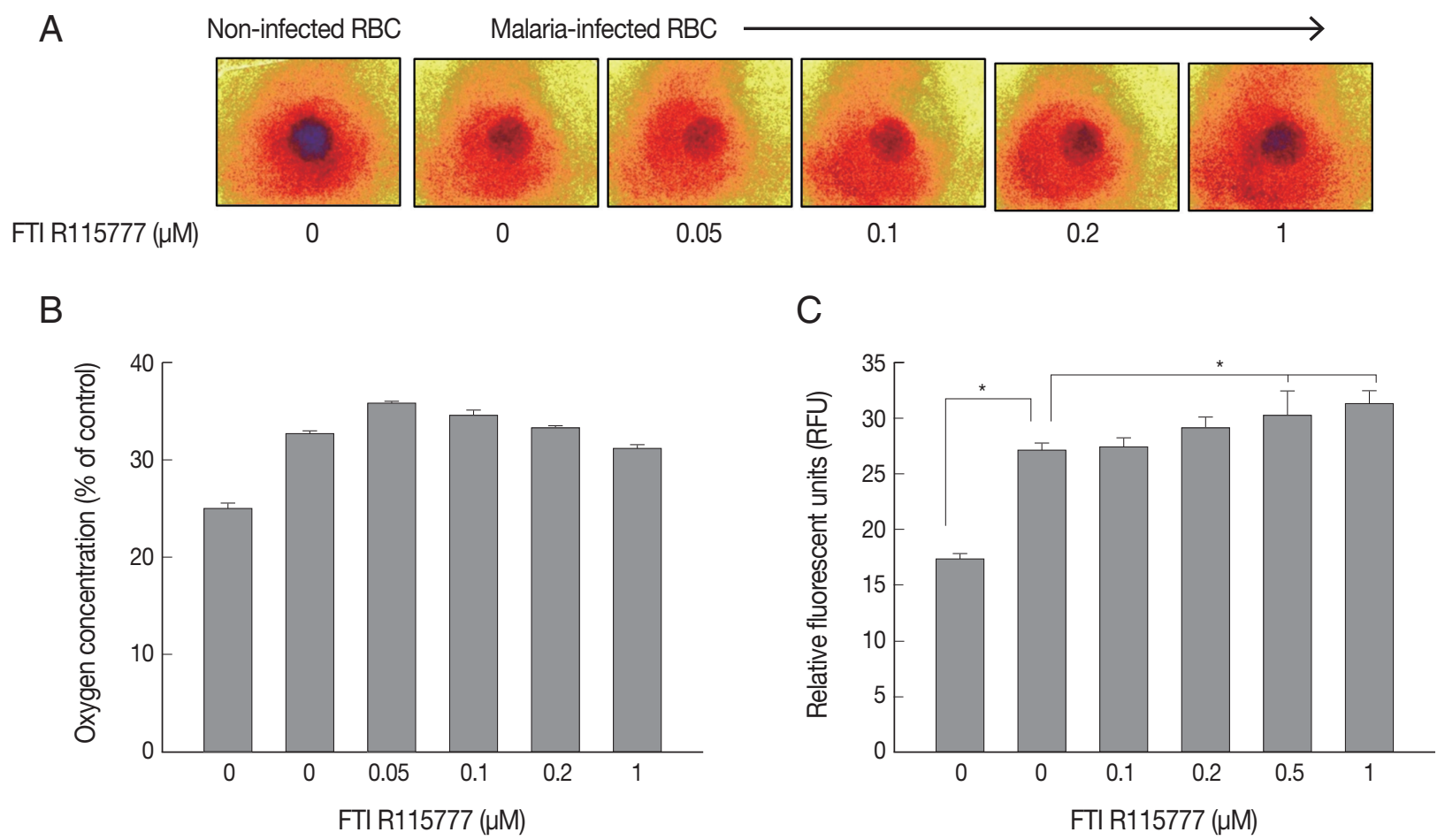

Fig. 3. Variations in oxygen concentration and ROS production in FTI R115777-treated P. falciparum. (A) Oxygen gradient of FTI R115777treated $P$. falciparum on the sensor foil. (B) Variation in oxygen level of FTI R115777-treated $P$. falciparum. (C) Variation in intercellular ROS level in FTI R115777-treated $P$. falciparum. Bars denote SD. ${ }^{\star} P<0.05$.

of mtDNA-encoded cytochrome $c$ oxidase III. The contents were dependent on the dose of FII R115777, as shown in Fig. $4 \mathrm{~A}$ and $\mathrm{B}$. Atovaquone and chloroquine have been used as mitochondrial specific inhibitor and general parasite inhibitor, respectively. The copy number of mitochondrial DNA-encoded cytochrome $c$ oxidase III was compared for chloroquine and atovaquone-treated $P$. falciparum. Chloroquine did not affect the copy number of mtDNA-encoded cytochrome $c$ oxidase III, while atovaquone increased the corresponding copy number (Fig. 4C-F). These results suggested that mtDNA-encoded cytochrome $c$ oxidase III of $P$. falciparum can be a potential target of FTI R115777.

\section{DISCUSSION}

FTIs are selective inhibitors of intracellular farnesyltransferase (FTase). FTIs are substrates for prenylation via FTase, including Ras, Pho-B, Rac, Pheb, nuclear lamins, and centromeric proteins, which interact with microtubules to promote the completion of mitosis [24]. FTase inhibition prevents the posttranslational processing of Ras proteins, which exert effects on multiple mechanisms of cellular survival, including angiogen- esis, cellular adhesion, and mitosis [24]. FTI R115777 (Zarnestra, Tipifarnib) is a nonpeptidomimetic FTI with clinical ability in some cancers, such as metastatic breast cancer, leukemia, and metastatic glioma [25]. A previous study demonstrated the cytotoxicity of FTIs to $P$. falciparum. The IC $_{50}$ of FTIs ranges from $0.7 \mathrm{nM}$ to $1,000 \mathrm{nM}$ in vitro [5]. Thus, FTI is a promising antimalarial agent. However, the target of FTIs in organelles such as mitochondria has not yet been investigated.

The mitochondrion in malaria parasites has a unique branched tricarboxylic acid metabolism without any activation of a pyruvate dehydrogenase. It does not show ATP production in blood stages of the parasite. A previous study has shown that mitochondrial electron transport chain of $P$. falciparum is involved in pyrimidine biosynthesis enzyme dihidroorotate dehydrogenase in blood stages [26]. In addition, it generates an electrochemical proton gradient across the inner membrane of mitochondrion that is utilized by the ATP synthase to drive the synthesis of ATP. The functional consequence of ATP synthase is interesting, because oxidative phosphorylation is not a major source of ATP in P. falciparum blood stages. Therefore, mitochondria of Plasmodium could be a target for antimalarial drugs [11]. Mitochondrial dysfunction can induce apoptotic 
A

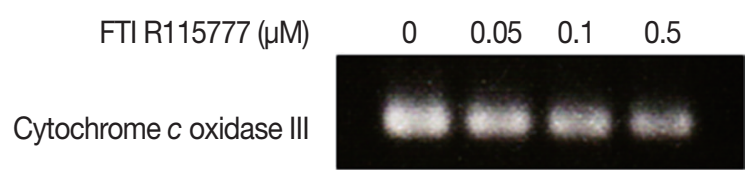

B

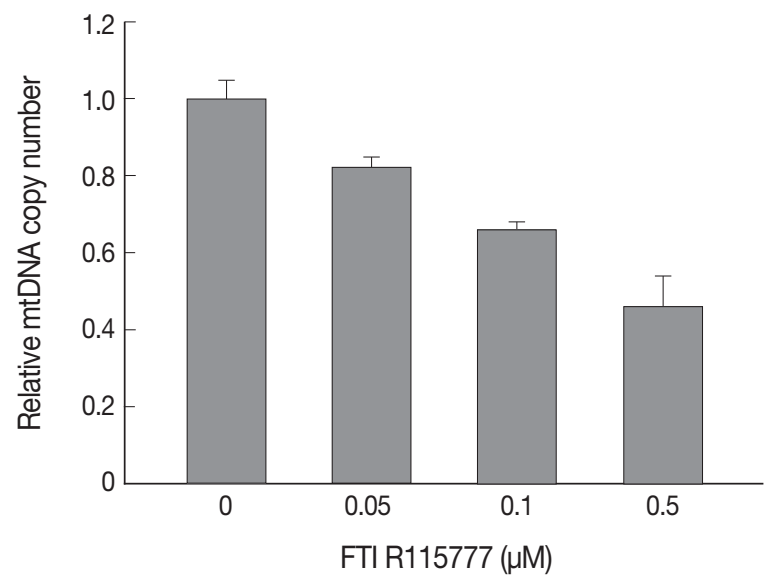

E

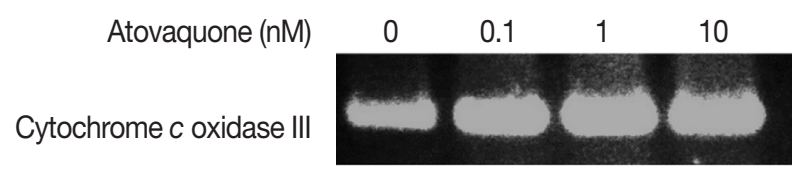

$\mathrm{F}$

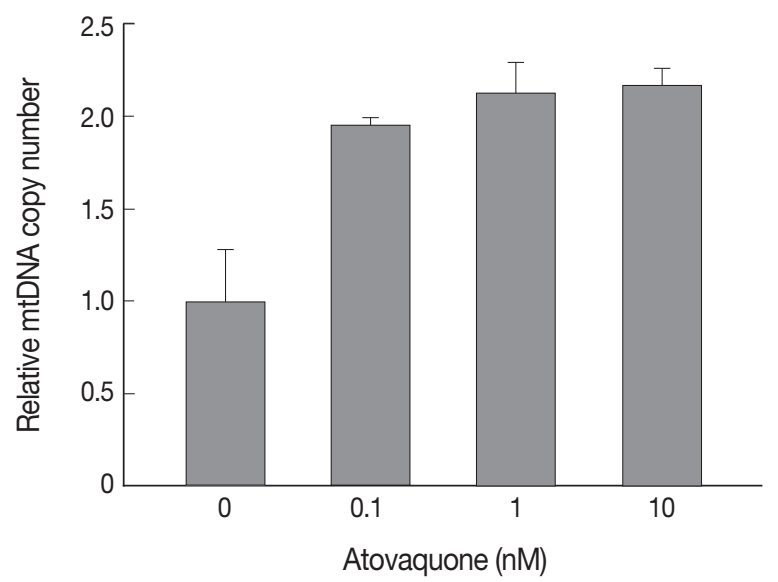

pathways [27]. The mitochondria control apoptosis at several levels, such as maintenance of ATP production and $\Delta \Psi \mathrm{m}$ and mitochondrial membrane permeability, to release certain apoptogenic factors from the intermembrane spaces into the cytosol [10]. Thus, $\Delta \Psi \mathrm{m}$ is a governing parameter of mitochondrial functions and cell health [27]. Changes in $\Delta \Psi \mathrm{m}$ are observed by staining with JC-1, a lipophilic cationic dye that selectively penetrates the mitochondria. This study shows that mitochondrial depolarization were induced by FTI R115777 dose
C

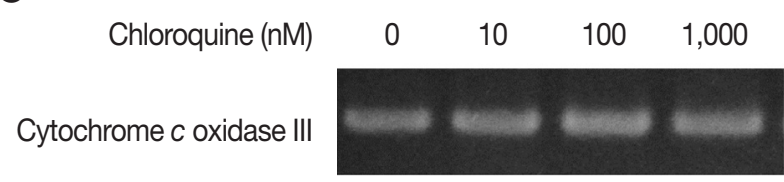

D

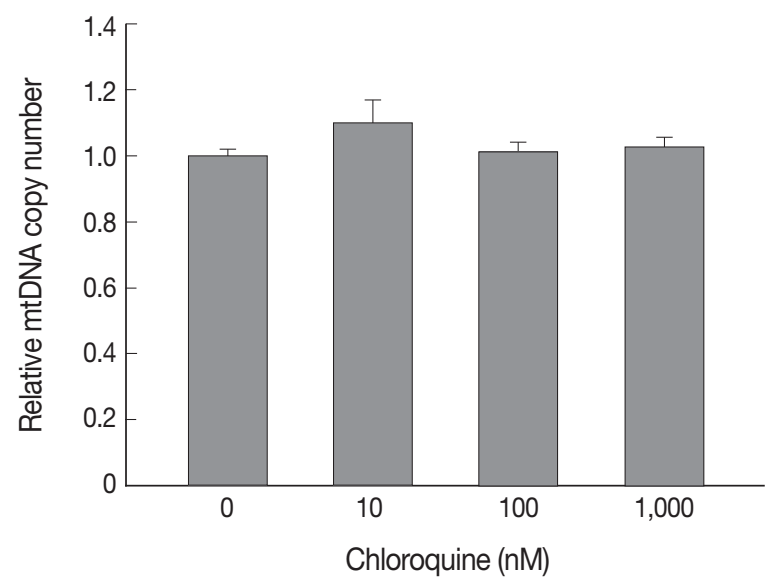

Fig. 4. Variation in copy number determined by PCR for mitochondrial cytochrome $c$ oxidase III. (A, B) Copy number of mtDNA cytochrome $c$ oxidase III in FTI R115777-treated P. falciparum. (C, D) Copy number of mtDNA cytochrome $c$ oxidase III in chloroquinetreated $P$. falciparum. (E, F) Copy number of mtDNA cytochrome $c$ oxidase III in atovaquone-treated $P$. falciparum.

dependently (Fig. 2).

The membrane potential generated by ATPase and mitochondrial electron transport chain (mETC) may be necessary because of the low oxygen consumption rate of $P$. falciparum. The decrease in ATP utilization inhibits oxygen consumption. Moreover, the respiration rate increases when ATP synthesis increases, thereby leading to the concept of respiratory control by ADP phosphorylation [28]. Oxygen is a terminal acceptor in the mitochondrial electron transport chain (mETC). Thus, the intra- 
cellular oxygen concentration has critical functions in physiological and pathological processes [29]. In a previous study, the extracellular and intracellular oxygen concentrations were assumed to be similar [29]. In the present study, we observed that the oxygen concentration of malaria-infected RBCs was higher than that of the non-infected RBCs. However, FTI R115777 did not affect the oxygen level in malaria-infected RBCs (Fig. 3A, B). The mitochondria depolarization by FII R115777 could be affected by other factors than oxygen consumption.

Mitochondria are crucial cellular energy-transducing organelles that function as a large source of ROS. ROS production can be controlled by $\Delta \Psi \mathrm{m}$. Dissipation of $\Delta \Psi \mathrm{m}$ leads to uncoupling of the electron transport chain [30]. ROS are required for mosquitoes to induce effective immune responses against Plasmodium [31]. Therefore, mitochondrial ROS are important activators in the immune response of the host to malaria infection [32]. The generation of ROS has been known to have a crucial function in the development of systemic complications in malaria [33]. In this study, ROS levels significantly increased in malariainfected RBCs than in non-infected RBCs. In addition, FTI R115777 induced the level of the intracellular ROS. This is true that FII R115777-induced intracellular ROS could sufficiently disrupt the mitochondrial functions. The specificity of FTI R115777 could be attributed to the selective interaction with malarial mitochondria. This interaction may specifically generate ROS in malarial mitochondria, which are key factors that enable the manifestation of the antimalarial drug effect of FII R115777.

The mitochondrial electron transport system can be used as a target for antimalarial drugs of $P$. falciparum [34]. In intra-erythrocytic malaria parasites, the mitochondrial electron transport system transfers the reducing equivalents generated by dihydroorotate dehydrogenase of the de novo pyrimidine biosynthetic pathway to ubiquinol-cytochrome $c$ reductase [34]. The cytochrome $c$ oxidase complex conducts the final step of electron transport in malaria parasites [35]. Plasmodium cytochrome $c$ oxidase has the most streamlined subunit composition among eukaryotes [36]. Thus, the expression of cytochrome $c$ oxidase represents the functions of Plasmodium. Cytochrome $c$ is released from mitochondria early in apoptosis before mitochondrial depolarization, activation of caspases, and DNA fragmentation [37].

Atovaquone which was introduced in the early stage of antimalarial drug development, has been known to inhibit the activity of cytochrome $c$ reductase in cholate-lysed mitochondria from P. falciparum and P. yoelii. It works on the cytochrome $b c_{1}$ complex in the malarial respiratory chain [38]. The role of mitochondria of malaria parasites is to dispose electrons generated by dihydroorotate dehydrogenase which is an essential enzyme in pyrimidine biosynthesis [38]. Therefore, the inhibition of dihydroorotate dehydrogenase has been suggested as antimalarial compounds, such as atovaquone [38].

In this study, we isolated the mitochondrial DNA and measured the copy number of mitochondrial DNA-encoded cytochrome $c$ oxidase from FTI R115777-treated $P$. falciparum. FTI R115777 inhibits the copy number of mitochondrial cytochrome $c$ oxidase dose dependently. mtDNA copy number were measured after treatment of chloroqunine and atovaquone as mitochondrial non-specific or specific inhibitor, respectively. FTI R115777 and atovaquone shows differences in the copy number of mtDNA-encoded cytochrome $c$ oxidase III. These drugs may act as a target of mitochondria through the distinguished mechanisms. To explain this different response of the copy number of mtDNA-encoded cytochrome $c$ oxidase III by FTI R115777 and atovaquone needs to be verified through a further study in the future.

The effect of FII R115777 on mitochondria of $P$. falciparum was in vitro analyzed in this study. The FTI R115777 was observed to inhibit the growth of malaria parasites effectively. It also affected the mitochondrial depolarization and ROS levels. The copy number of mtDNA-encoded cytochrome $c$ oxidase III was significantly reduced by FTI R115777. The experimental data obtained in this study supported that FTI R115777 has strong influence on the mitochondria of $P$. falciparum by deteriorating the function of malarial mitochondria.

\section{ACKNOWLEDGMENT}

This work was supported by the National Research Foundation of Korea grant funded by the Korea government (MSIP) (no. 2008-0061991).

\section{CONFLICT OF INTEREST}

The authors declare no competing interests.

\section{REFERENCES}

1. Farooq U, Mahajan RC. Drug resistance in malaria. J Vector Borne Dis 2004; 41: 45-53.

2. Hogg T, Nagarajan K, Herzberg S, Chen L, Shen X, Jiang H, 
Wecke M, Blohmke C, Hilgenfeld R, Schmidt CL. Structural and functional characterization of falcipain-2, a hemoglobinase from the malarial parasite Plasmodium falciparum. J Biol Chem 2006; 281: 25425-25437.

3. Sidhu AB, Verdier-Pinard D, Fidock DA. Chloroquine resistance in Plasmodium falciparum malaria parasites conferred by pfort mutations. Science 2002; 298: 210-213.

4. Wang J, Huang L, Li J, Fan Q, Long Y, Li Y, Zhou B. Artemisinin directly targets malarial mitochondria through its specific mitochondrial activation. PLoS One 2010; 5: e9582.

5. Nallan L, Bauer KD, Bendale P, Rivas K, Yokoyama K, Horney CP, Pendyala PR, Floyd D, Lombardo LJ, Williams DK, Hamilton A, Sebti S, Windsor WT, Weber PC, Buckner FS, Chakrabarti D, Gelb MH, Van Voorhis WC. Protein farnesyltransferase inhibitors exhibit potent antimalarial activity. J Med Chem 2005; 48 : 3704-3713.

6. Vigil D, Cherfils J, Rossman KL, Der CJ. Ras superfamily GEFs and GAPs: validated and tractable targets for cancer therapy? Nat Rev Cancer 2010; 10: 842-857.

7. Wiesner J, Kettler K, Sakowski J, Ortmann R, Katzin AM, Kimura EA, Silber K, Klebe G, Jomaa H, Schlitzer M. Farnesyltransferase inhibitors inhibit the growth of malaria parasites in vitro and in vivo. Angew Chem Int Ed Engl 2004; 43: 251-254.

8. Appels NM, Beijnen JH, Schellens JH. Development of farnesyl transferase inhibitors: a review. Oncologist 2005; 10: 565-578.

9. Kohring K, Wiesner J, Altenkamper M, Sakowski J, Silber K, Hillebrecht A, Haebel P, Dahse HM, Ortmann R, Jomaa H, Klebe G, Schlitzer M. Development of benzophenone-based farnesyltransferase inhibitors as novel antimalarials. ChemMedChem 2008; 3: 1217-1231.

10. Ly JD, Grubb DR, Lawen A. The mitochondrial membrane potential (deltapsi(m)) in apoptosis; an update. Apoptosis 2003; 8: 115-128.

11. Vaidya $A B$, Mather MW. Mitochondrial evolution and functions in malaria parasites. Annu Rev Microbiol 2009; 63: 249-267.

12. Torrentino-Madamet M, Desplans J, Travaille C, James Y, Parzy D. Microaerophilic respiratory metabolism of Plasmodium falciparum mitochondrion as a drug target. Curr Mol Med 2010; 10 : 29-46.

13. Trager W, Jensen JB. Human malaria parasites in continuous culture. Science 1976; 193: 673-675.

14. Lambros C, Vanderberg JP. Synchronization of Plasmodium falciparum erythrocytic stages in culture. J Parasitol 1979; 65: 418420 .

15. Salehnia M, Tohonen V, Zavareh S, Inzunza J. Does cryopreservation of ovarian tissue affect the distribution and function of germinal vesicle oocytes mitochondria? Biomed Res Int 2013; 2013: 8 .

16. Kellner K, Liebsch G, Klimant I, Wolfbeis OS, Blunk T, Schulz MB, Gopferich A. Determination of oxygen gradients in engineered tissue using a fluorescent sensor. Biotechnol Bioeng 2002; 80: 73-83.

17. Othman A, Ahmad S, Megyerdi S, Mussell R, Choksi K, Mad- dipati KR, Elmarakby A, Rizk N, Al-Shabrawey M. 12/15-Lipoxygenase-derived lipid metabolites induce retinal endothelial cell barrier dysfunction: contribution of NADPH oxidase. PLoS One 2013; 8: e57254.

18. Hamilton ML, Van Remmen H, Drake JA, Yang H, Guo ZM, Kewitt K, Walter CA, Richardson A. Does oxidative damage to DNA increase with age? Proc Natl Acad Sci USA 2001; 98: 10469-10474.

19. Cunha MG, Medina TS, Oliveira SG, Marinho AN, Povoa MM, Ribeiro-dos-Santos AK. Development of a polymerase chain reaction (PCR) method based on amplification of mitochondrial DNA to detect Plasmodium falciparum and Plasmodium vivax. Acta Trop 2009; 111: 35-38.

20. Buckling A, Ranford-Cartwright LC, Miles A, Read AF. Chloroquine increases Plasmodium falciparum gametocytogenesis in vitro. Parasitology 1999; 118 (Part 4): 339-346.

21. Painter HJ, Morrisey JM, Mather MW, Vaidya AB. Specific role of mitochondrial electron transport in blood-stage Plasmodium falciparum. Nature 2007; 446: 88-91.

22. Narendra DP, Jin SM, Tanaka A, Suen DF, Gautier CA, Shen J, Cookson MR, Youle RJ. PINK1 is selectively stabilized on impaired mitochondria to activate parkin. PLoS Biol 2010; 8: e1000298.

23. Chazotte B. Labeling mitochondria with MitoTracker dyes. Cold Spring Harb Protoc 2011; 2011: 990-992.

24. Lancet JE, Gojo I, Gotlib J, Feldman EJ, Greer J, Liesveld JL, Bruzek LM, Morris L, Park Y, Adjei AA, Kaufmann SH, Garrett-Mayer E, Greenberg PL, Wright JJ, Karp JE. A phase 2 study of the farnesyltransferase inhibitor tipifarnib in poor-risk and elderly patients with previously untreated acute myelogenous leukemia. Blood 2007; 109: 1387-1394.

25. Zhu KC, Gerbino E, Beaupre DM, Mackley PA, Muro-Cacho C, Beam C, Hamilton AD, Lichtenheld MG, Kerr WG, Dalton W, Alsina M, Sebti SM. Farnesyltransferase inhibitor R115777 (Zarnestra, Tipifarnib) synergizes with paclitaxel to induce apoptosis and mitotic arrest and to inhibit tumor growth of multiple myeloma cells. Blood 2005; 105: 4759-4766.

26. Balabaskaran Nina P, Morrisey JM, Ganesan SM, Ke H, Pershing AM, Mather MW, Vaidya AB. ATP synthase complex of Plasmodium falciparum: dimeric assembly in mitochondrial membranes and resistance to genetic disruption. J Biol Chem 2011; 286: 41312-41322.

27. Kataoka M, Fukura Y, Shinohara Y, Baba Y. Analysis of mitochondrial membrane potential in the cells by microchip flow cytometry. Electrophoresis 2005; 26: 3025-3031.

28. Rousset S, Alves-Guerra MC, Mozo J, Miroux B, Cassard-Doulcier AM, Bouillaud F, Ricquier D. The biology of mitochondrial uncoupling proteins. Diabetes 2004; 53 (suppl 1): S130-S135.

29. Shen J, Khan N, Lewis LD, Armand R, Grinberg O, Demidenko E, Swartz H. Oxygen consumption rates and oxygen concentration in molt- 4 cells and their mtDNA depleted (rho0) mutants. Biophys J 2003; 84: 1291-1398.

30. Goncalves RL, Oliveira JH, Oliveira GA, Andersen JF, Oliveira 
MF, Oliveira PL, Barillas-Mury C. Mitochondrial reactive oxygen species modulate mosquito susceptibility to Plasmodium infection. PLoS One 2012; 7: e41083.

31. Molina-Cruz A, DeJong RJ, Charles B, Gupta L, Kumar S, Jaramillo-Gutierrez G, Barillas-Mury C. Reactive oxygen species modulate Anopheles gambiae immunity against bacteria and Plasmodium. J Biol Chem 2008; 283: 3217-3223.

32. West AP, Brodsky IE, Rahner C, Woo DK, Erdjument-Bromage H, Tempst P, Walsh MC, Choi Y, Shadel GS, Ghosh S. TLR signalling augments macrophage bactericidal activity through mitochondrial ROS. Nature 2011; 472: 476-480.

33. Pabon A, Carmona J, Burgos LC, Blair S. Oxidative stress in patients with non-complicated malaria. Clin Biochem 2003; 36: 71-78.

34. Krungkrai J, Krungkrai SR, Suraveratum N, Prapunwattana P. Mitochondrial ubiquinol-cytochrome $c$ reductase and cytochrome $c$ oxidase: chemotherapeutic targets in malarial parasites. Biochem Mol Biol Int 1997; 42: 1007-1014.

35. Gardner MJ, Hall N, Fung E, White O, Berriman M, Hyman RW,
Carlton JM, Pain A, Nelson KE, Bowman S, Paulsen IT, James K, Eisen JA, Rutherford K, Salzberg SL, Craig A, Kyes S, Chan MS, Nene V, Shallom SJ, Suh B, Peterson J, Angiuoli S, Pertea M, Allen J, Selengut J, Haft D, Mather MW, Vaidya AB, Martin DM, Fairlamb AH, Fraunholz MJ, Roos DS, Ralph SA, McFadden GI, Cummings LM, Subramanian GM, Mungall C, Venter JC, Carucci DJ, Hoffman SL, Newbold C, Davis RW, Fraser CM, Barrell B. Genome sequence of the human malaria parasite Plasmodium falciparum. Nature 2002; 419: 498-511.

36. Sullivan SKD. Malaria: Drugs, Disease and Post-gemonic Biology. New York, USA. Springer. 2010.

37. Yang J, Liu X, Bhalla K, Kim CN, Ibrado AM, Cai J, Peng TI, Jones DP, Wang X. Prevention of apoptosis by Bcl-2: release of cytochrome c from mitochondria blocked. Science 1997; 275: 11291132.

38. Srivastava IK, Rottenberg H, Vaidya AB. Atovaquone, a broad spectrum antiparasitic drug, collapses mitochondrial membrane potential in a malarial parasite. J Biol Chem 1997; 272: 39613966. 